\title{
Coherent Resonant X-Ray Scattering from a Rotating Medium
}

\author{
R. Röhlsberger, ${ }^{1 *}$ T. S. Toellner, ${ }^{2}$ W. Sturhahn,${ }^{2}$ K. W. Quast,${ }^{2}$ E. E. Alp, ${ }^{2}$ A. Bernhard, ${ }^{1}$ E. Burkel,${ }^{1}$ \\ O. Leupold, ${ }^{3}$ and E. Gerdau ${ }^{3}$ \\ ${ }^{1}$ Universität Rostock, Fachbereich Physik, August-Bebel-Strasse 55, 18055 Rostock, Germany \\ ${ }^{2}$ Advanced Photon Source, Argonne National Laboratory, Argonne, Illinois 60439 \\ ${ }^{3}$ II. Institut für Experimentalphysik, Luruper Chaussee 149, 22761 Hamburg, Germany
}

(Received 5 August 1999)

\begin{abstract}
A coherently excited nuclear state in a rotating sample acquires a phase shift during its time evolution that is proportional to its angular momentum and the rotation angle. As a consequence, the radiative decay of the excited state proceeds into the rotated direction, and the time spectrum of the nuclear decay is mapped onto an angular scale. This effect has been observed in nuclear resonant scattering of synchrotron radiation from a ${ }^{57} \mathrm{Fe}$ metal foil rotating at $18 \mathrm{kHz}$.
\end{abstract}

PACS numbers: 76.80. $+\mathrm{y}, 42.50 . \mathrm{Ct}, 78.70 . \mathrm{Ck}$

Quantum objects like photons or neutrons acquire dynamical phase shifts upon propagation in rotating frames that can be measured in interferometry or diffraction experiments. Such phase shifts were first demonstrated for photons in a rotating optical interferometer [1] and then also observed via neutron interferometry in rotating frames, see, e.g., [2]. The occurrence of such interference effects requires the transit time of the particle through the system to be sufficiently long for the phase shifts to evolve. In the above examples this is ensured through the finite time of flight of the particle in the rotating system. Another possibility to fulfill this condition is the excitation of a resonance in the rotating system with an energy width $\Gamma_{0}$. The propagation of particles in the system is then affected by resonant scattering processes that take place on a time scale given by $\tau=\hbar / \Gamma_{0}$. If the resonance is excited by a radiation pulse that is short compared to $\tau$, a collectively excited state (exciton) is created, where a single excitation is coherently distributed over the atoms of the sample [3]. The coherence leads to remarkable properties of the exciton decay, notably the speedup of the radiative decay compared to that of an isolated atom, and quantum beats resulting from the interference of waves emitted from different resonances at different atoms. So far, such exciton states have been prepared by short-pulse laser radiation exciting long-lived electronic levels $[4,5]$ or by synchrotron radiation pulses recoilessly exciting nuclear levels [6]. The analysis of the temporal evolution of the subsequent radiative decay provides valuable information about the environment of the atoms in the sample. Therefore, this type of time-resolved spectroscopy has generated several applications in condensed matter physics $[5,6]$.

In this Letter, we describe an effect that arises due to the evolution of dynamical phases upon nuclear resonant scattering in rotating frames. The excitation of a system of nuclei by a synchrotron radiation pulse creates a phased nuclear exciton state with wave vector $\vec{k}_{0}$ [3], causing the subsequent radiative decay to proceed in the forward direction. This established the technique of nuclear resonant forward scattering (NFS) of synchrotron radiation as the time-based analog of the Mössbauer effect [7]. In a rotating system the spatial phases of the nuclei are subject to a well-defined time evolution. If their relative phases are preserved during the nuclear lifetime, one intuitively expects the exciton to be rotated as a whole. Consequently, the direction of the radiative decay should deviate from the direction of $\vec{k}_{0}$ by the rotation angle that has developed during the lifetime of the excited state. As a result, the time spectrum of the nuclear decay is mapped to an angular scale, as sketched in Fig. 1.

For an experimental test of this prediction, we have employed nuclear resonant scattering at the $14.4 \mathrm{keV}$ resonance of ${ }^{57} \mathrm{Fe}$ with a level width of $\Gamma_{0}=4.7 \mathrm{neV}$ and a lifetime of $\tau=141 \mathrm{~ns}$. The hyperfine splitting of the nuclear levels in a ferromagnetic environment leads to quantum-beat periods of several ns in the time-resolved nuclear decay. To obtain an angular separation of, say, $0.1 \mathrm{mrad}$ for events that are separated by $1 \mathrm{~ns}$, rotational speeds in

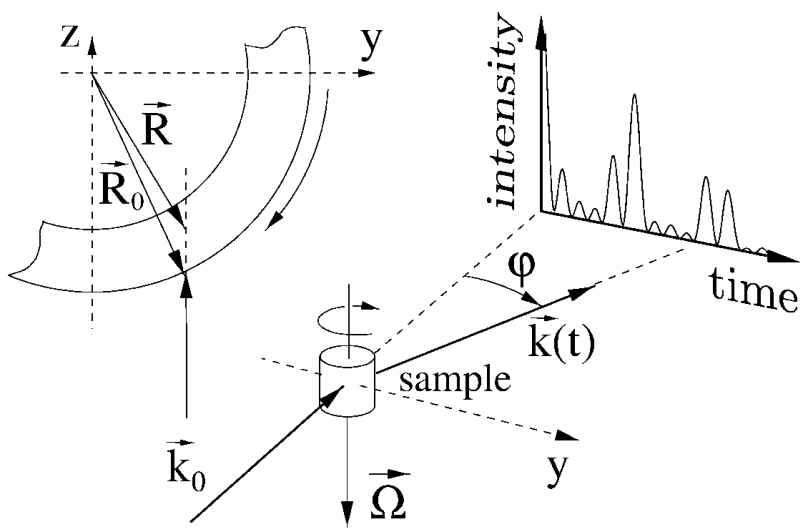

FIG. 1. Scattering geometry of nuclear resonant scattering at a sample rotating with angular velocity $\vec{\Omega}$. The time spectrum of the radiative nuclear decay is mapped to an angular scale. An off-center displacement of the incident wave vector does not affect the angular mapping, but leads to an energy shift of the scattered radiation, as explained below. 
the order of $10 \mathrm{kHz}$ are necessary. Such high-speed rotary motion is commonly used in the magic-angle-spinning (MAS) technique of solid state NMR [8] with frequencies up to $35 \mathrm{kHz}$.

The experiment was performed at the undulator beam line 3-ID at the Advanced Photon Source. The experimental setup is shown schematically in Fig. 2. The radiation was monochromatized to a bandwidth of $1 \mathrm{eV}$ by a water-cooled high-heat-load monochromator employing two symmetric diamond (111) reflections. A subsequent slit system was adjusted to define a beam cross section of $500 \times 30 \mu \mathrm{m}^{2}$ (horizontal $\times$ vertical), resulting in a typical flux of $2 \times 10^{10} \mathrm{~s}^{-1}$ on the sample. For sample rotation, a commercially available NMR-MAS system was used (Bruker Analytical GmbH, Germany), slightly modified for $\mathrm{x}$-ray transmission. The samples were contained in hollow cylinders ( $4 \mathrm{~mm}$ diameter, $0.5 \mathrm{~mm}$ wall thickness) consisting of sintered polycrystalline $\mathrm{Si}_{3} \mathrm{~N}_{4}$. After transmission through the rotor, the beam was guided through an evacuated flight tube to minimize small-angle scattering in air. The detector assembly consisted of an avalanche photodiode with an aperture of the same cross section as defined by the upstream slit system. It was used in a distance of $3.0 \mathrm{~m}$ downstream of the rotor in vertical-scan mode to record the distribution of the radiation scattered from the rotor. No time discrimination as typically used in the NFS technique was applied. The sample was a $5.7 \mu \mathrm{m}$ thick foil of polycrystalline $\alpha-{ }^{57} \mathrm{Fe}$ that covered less than one-half of the inner circumference of the rotor (cf. Fig. 2). This was done to avoid interference effects resulting from the beam passing the resonant absorber twice. The sample was spun around a horizontal axis with a rotational frequency of $18 \mathrm{kHz}$, corresponding to an angular velocity of $0.11 \mathrm{mrad} / \mathrm{ns}$. The angular distribution of the scattered radiation was recorded up to a scattering angle of $10 \mathrm{mrad}$ relative to the primary beam. The data are shown in Fig. 3. Quantum beats of nuclear resonant scattering with a separation of $1-1.5 \mathrm{mrad}$ are clearly visible on top of a background (dotted line) that mainly results from small-angle scattering at the rotor material. This observation qualitatively confirms that the time response of nuclear resonant

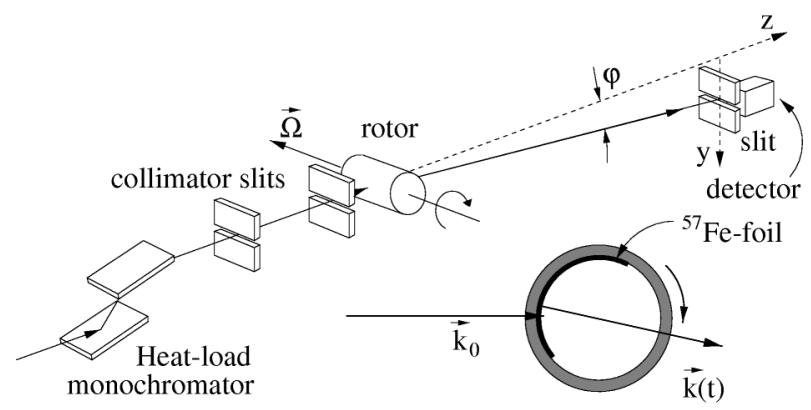

FIG. 2. Scheme of the experimental setup. The scattered radiation from the rotor was guided through an evacuated flight tube to the detector. The coordinates refer to the same frame as introduced in Fig. 1. In the bottom right a cross sectional view of the scattering at the rotor is shown. scattering in rotating frames is mapped to an angular scale [10]. The apparent analogy with a sweeping ray of light suggests the name "nuclear lighthouse effect."

For a theoretical description of this effect we calculate the propagation of radiation through a homogeneous ensemble of resonant nuclei. In a space-time formulation of this problem the amplitude in depth $z=\left|\vec{R}-\vec{R}_{0}\right|$ of the sample at time $t$ is given by [11]

$$
\vec{A}(z, t)=\exp \left[-i z \int_{-\infty}^{t} d t^{\prime} \mathbf{N}\left(t, t^{\prime}\right)\right] \vec{A}_{0}\left(t^{\prime}\right) .
$$

In case of nuclear resonant scattering as a second-order process the kernel $\mathbf{N}\left(t, t^{\prime}\right)$ results from the evaluation of a two-point correlation function in space-time [12]. It describes the evolution of the atom and the radiation field between the time of excitation and deexitation, $t^{\prime}$ and $t$, respectively. Boldface capital letters shall denote matrix-valued quantities to account for the polarization dependence of the scattering. In experiments with pulsed synchrotron radiation, the incident radiation field is given to a very good approximation by $\vec{A}_{0}(t)=\delta(t) \vec{A}_{0}$. This facilitates to divide the solution of Eq. (1) into a prompt pulse that has passed the sample without resonant interaction and the delayed response from the collectively excited ensemble of nuclei,

$$
\vec{A}(z, t)=[\mathbf{1} \delta(t)-\mathbf{G}(z, t)] \vec{A}_{0},
$$

where 1 is the unit matrix. For the evaluation of Eq. (1) in case of a rotating sample we have to determine the transformation of the kernel $\mathbf{N}$ from the center-of-mass system of the rotating nucleus into a fixed laboratory frame. The result is [13]

$$
\mathbf{N}\left(t, t^{\prime}\right)=\mathbf{M}_{-}(\vec{R}, t) \mathbf{N}_{0}\left(t, t^{\prime}\right) \mathbf{M}_{+}\left(\vec{R}, t^{\prime}\right) .
$$

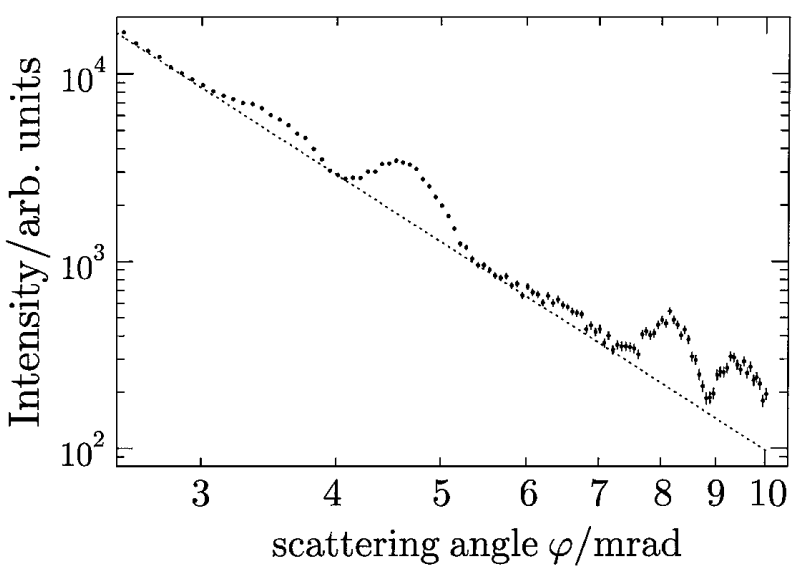

FIG. 3. Measured angular distribution of $14.4 \mathrm{keV}$ photons scattered from a $5.7 \mu \mathrm{m}$ thick foil of ${ }^{57} \mathrm{Fe}$ spinning at $18 \mathrm{kHz}$. The resonantly scattered quanta appear on top of a background that mainly results from small-angle $\mathrm{x}$-ray scattering (SAX) at the rotor material. SAX of the resonantly scattered radiation itself can be neglected here. The angular distribution of the SAX was measured independently and fitted with a $\varphi^{-3.89}$ dependence (dotted line) that is close to Porod's law for the structure factor of randomly oriented particles [9]. 
The functions $\mathbf{M}_{ \pm}(\vec{R}, t)$ are expectation values of the operator that generates the motion of the nuclei. In case of rotary motion with constant angular velocity $\vec{\Omega}$ they are given by

$$
\mathbf{M}_{ \pm}(\vec{R}, t)=\left\langle\gamma\left|e^{ \pm i \vec{\Omega} \cdot \vec{J} t}\right| \gamma\right\rangle e^{ \pm i \overrightarrow{k_{0}} \cdot \vec{R}}
$$

where $\vec{R}$ is the position vector of the nucleus relative to the center of rotation, as sketched in Fig. 1 [14]. $\overrightarrow{\mathbf{J}}=$ $\vec{R} \times \overrightarrow{\mathbf{P}}+\overrightarrow{\mathbf{S}}$ is the operator of the total angular momentum of the system, including the electromagnetic field, consisting of orbital and spin angular momentum. $\overrightarrow{\mathbf{P}}$ is the operator of the total linear momentum of the system. For the photon state vectors we assume $|\gamma\rangle=\mathbf{a}_{k_{0}, \epsilon}^{\dagger}|0\rangle$, where $\mathbf{a}_{k_{0}, \epsilon}^{\dagger}$ is a creation operator of the photon field with wave vector $\vec{k}_{0}$ and polarization $\epsilon$, and $|0\rangle$ represents the electromagnetic vacuum. This ansatz contains the assumption that the radiation field does not contain more than one photon per mode, which is a good approximation even for present-day high-brilliance synchrotron radiation sources. Neglecting the action of the spin operator $\overrightarrow{\mathbf{S}}$ on the state vectors [15], the evaluation of Eq. (4) yields

$$
\mathbf{M}_{ \pm}(\vec{R}, t)=\mathbf{1} e^{ \pm i \vec{k}(t) \cdot \vec{R}},
$$

with

$$
\vec{k}(t)=\vec{k}_{0}-\vec{k}_{0} \times \vec{\Omega} t+\frac{1}{2}\left(\vec{k}_{0} \times \vec{\Omega}\right) \times \vec{\Omega} t^{2}-\cdots
$$

Inserting this into Eq. (3), the scattered amplitude according to Eq. (1) is given by [16]

$$
\vec{A}(z, t)=\left[\mathbf{1} \delta(t)-\mathbf{M}_{-}(\vec{R}, t) \mathbf{G}_{0}(z, t) \mathbf{M}_{+}(\vec{R}, 0)\right] \vec{A}_{0} .
$$

Evaluation of this result and comparison with Eq. (2) results in the following expression for the delayed response of the rotating nuclear ensemble:

$$
\mathbf{G}(z, t)=\mathbf{G}_{0}(z, t) e^{-i\left(\vec{k}(t)-\vec{k}_{0}\right) \cdot \vec{R}},
$$

where $\mathbf{G}_{0}(z, t)$ denotes the delayed time response from the sample at rest. The phase factor indicates that the nuclear exciton is carried with the rotating sample during its lifetime and that its radiative decay proceeds into the rotated direction given by $\vec{k}(t)$. Note that the first-order term in the expansion of $\vec{k}(t) \cdot \vec{R}$ implies that the scattering process takes place at an energy that is Doppler shifted by $\Delta E=-\hbar\left(\vec{k}_{0} \times \vec{\Omega}\right) \cdot \vec{R}=-\hbar \vec{v} \cdot \vec{k}_{0}$ where $\vec{v}$ is the instantaneous linear velocity of the rotating sample. Thus, at sufficiently high rotational speeds the deflected radiation can be tuned over a range of several meV which may provide the basis for $\mathrm{x}$-ray spectroscopy with $\mu \mathrm{eV}$ resolution [17].

The mapping to an angular scale is described most conveniently by a one-dimensional transformation into reciprocal space based on the geometry shown in Fig. 1. In this step we have to consider that the incident radiation is not a plane wave but a superposition of waves spread over a range of directions. This leads to a finite degree of transverse coherence at the sample position that is described by the mutual coherence function $W(y)$ [18]. Taking the phase factor in Eq. (8) up to first order in $t$, the delayed response in reciprocal space is then given by

$$
\mathbf{G}(\vec{q}, t)=\mathbf{G}_{0}(t) \int e^{-i\left(\vec{q}-\vec{k}_{0} \times \vec{\Omega} t\right) \cdot \vec{R}} W(y) d y .
$$

$W(y)$ is often represented by a Gaussian with standard deviation $\xi$, which is then taken as a definition of the transverse coherence length. Based on the geometry sketched in Fig. 1 we now write $|\vec{q}|=k_{0} \varphi$ and $\left(\vec{k}_{0} \times \vec{\Omega}\right) \cdot \vec{R}=$ $-k_{0} \Omega y$. Evaluation of Eq. (9) then leads to the following expression for the scattered intensity as a function of angular coordinate $\varphi$ only (integrating over all times):

$$
I(\varphi)=\int_{0}^{\infty}\left|\mathbf{G}_{0}(t) \vec{A}_{0}\right|^{2} e^{-k_{0}^{2} \xi^{2} \Omega^{2}(t-\varphi / \Omega)^{2}} d t .
$$

Thus the effect of the finite degree of transverse coherence is to introduce a resolution function as represented by the Gaussian in the above equation. If the half-width of this function, given by $\Delta t=2 \sqrt{\ln 2} /\left(k_{0} \xi \Omega\right)$ is much smaller than the typical quantum-beat period to be resolved, Eq. (10) can be well approximated by

$$
I(\varphi)=\left|\mathbf{G}_{0}(\varphi / \Omega) \vec{A}_{0}\right|^{2} .
$$

This result expresses the direct mapping of the time scale to an angular scale as described by $t=\varphi / \Omega$. It allows one to evaluate the angular distribution of the scattered intensity according to the theory of time-resolved nuclear forward scattering.

The angular distribution of resonant scattering from the rotating ${ }^{57} \mathrm{Fe}$ foil, obtained after background subtraction of the data in Fig. 3, is shown in Fig. 4. In this experiment, the transverse coherence length was $\xi \approx 16 \mu \mathrm{m}$ [19] at the position of the rotor, leading to a value of $\Delta t \approx 12 \mathrm{ps}$ for the width of the resolution function at a rotational frequency of $18 \mathrm{kHz}$. This estimate justifies to apply Eq. (11) for the evaluation of the data. The result of the theoretical fit is shown in Fig. 4 as a solid line. In the inset, we show a time spectrum of nuclear resonant forward scattering that was measured with the foil at rest using the conventional timing technique. The agreement between both sets of data confirms that the radiative nuclear decay proceeds on an angular scale according to $\varphi=\Omega t$. The nuclear lighthouse effect thus allows one to obtain time spectra of nuclear resonant scattering by purely geometrical means. The temporal evolution of nuclear resonant scattering can be conveniently recorded as a function of a spatial coordinate only. As a result, this effect can be exploited in cases where the conventional timing technique is difficult, or impossible, to apply. For example, time resolutions can be achieved that are not possible with existing x-ray detectors. At a rotational frequency of $35 \mathrm{kHz}$ an effective time resolution in the range of $20 \mathrm{ps}$ can be achieved at present-day synchrotron radiation sources. Since the magnitude of the angular deflection depends only on the 


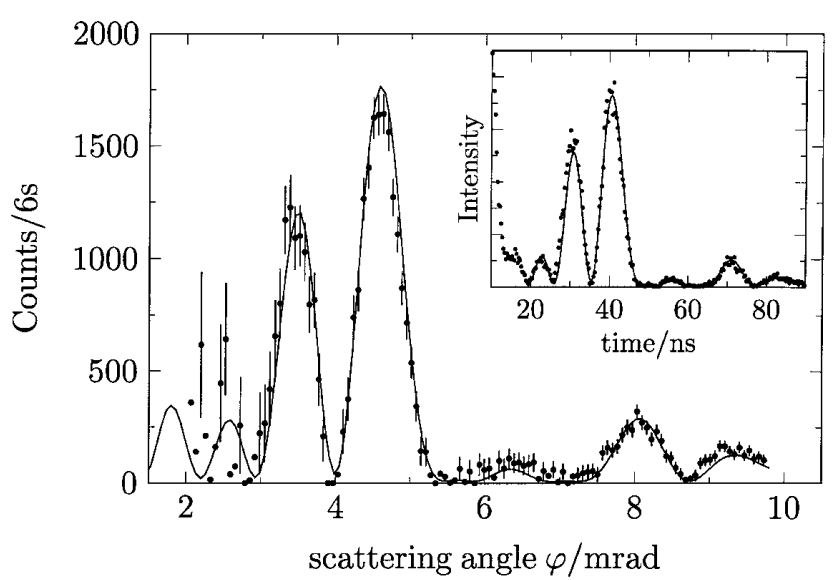

FIG. 4. Angular distribution of the radiation scattered from the $5.7 \mu \mathrm{m}{ }^{57} \mathrm{Fe}$ foil rotating at $18 \mathrm{kHz}$, obtained after the background subtraction of the data in Fig. 3. The solid line is a fit according to Eq. (11). The inset shows the data of time-resolved nuclear forward scattering from the foil at rest.

time difference between excitation and reemission, this effect is independent of the time structure of the exciting radiation. Therefore, the achievable time resolution is not limited by the electron bunch length as it is in the case of the conventional timing technique. Possible applications are the investigation of samples with very short lifetimes or very large nuclear level splittings. For example, quantum-beat periods below $100 \mathrm{ps}$ are expected in the coherent decay of the $25.65 \mathrm{keV}$ resonance of ${ }^{161} \mathrm{Dy}$ in the presence of magnetic ordering. The technique can be easily extended to other isotopes without the need for high-resolution crystal optics, as has been demonstrated in the cases of ${ }^{119} \mathrm{Sn}$ and ${ }^{149} \mathrm{Sm} \mathrm{[20].} \mathrm{The} \mathrm{small-an-}$ gle scattering background can be significantly reduced by the use of a high-resolution monochromator with an energy bandwidth of a few $\mathrm{meV}$ [21] and/or the use of a rotor consisting of single-crystalline material like sapphire. Then the time spectra of the nuclear decay can be recorded with a position-sensitive detector like an image plate.

In conclusion, we have observed an effect that occurs in nuclear resonant scattering of synchrotron radiation in rotating frames. Based on the transformation properties of the scattering function under rotations we have derived that resonantly scattered radiation is deflected by an angle that is proportional to the decay time of the intermediate excited state. Our theoretical description suggests that this effect is not restricted to nuclear resonances, but may be observed in other systems possessing excited states with sufficiently long lifetimes.

We gratefully acknowledge the assistance of P. Lee and M. Hu during the experiments. R. R. acknowledges stimulating discussions with U. Bergmann and S. Ruby. This work was supported by the German BMBF under Contract No. 05 643HRA 5. Use of the Advanced Photon Source was supported by the U.S. Department of Energy, Basic Energy Sciences, Office of Science, under Contract No. W-31-109-ENG-38.

*Electronic mail: roehle@physik1.uni-rostock.de

[1] M. G. Sagnac, C.R. Acad. Sci. 157, 708 (1913); 157, 1410 (1913).

[2] D. K. Atwood, M. A. Horne, C. G. Shull, and J. Arthur, Phys. Rev. Lett. 52, 1673 (1984).

[3] J.P. Hannon and G. T. Trammell, Physica (Amsterdam) 159B, 161 (1989).

[4] S. Haroche, in High Resolution Laser Spectroscopy, edited by K. Shimoda (Springer, Berlin, 1976).

[5] H. Stolz, Time Resolved Light Scattering from Excitons (Springer, Berlin, 1993).

[6] Nuclear Resonant Scattering of Synchrotron Radiation, edited by E. Gerdau and H. de Waard (Baltzer Science Publishers, Oxford, 1999).

[7] J. B. Hastings, D. P. Siddons, U. van Bürck, R. Hollatz, and U. Bergmann, Phys. Rev. Lett. 66, 770 (1991).

[8] F. D. Doty and P.D. Ellis, Rev. Sci. Instrum. 52, 1868 (1981).

[9] See, e.g., A. Guinier and G. Fournet, Small-Angle Scattering of X-Rays (John Wiley, New York, 1955).

[10] Signs for an angular shift of radiation resonantly scattered from a rotating sample were observed already at considerably lower rotational frequencies; see [17,22].

[11] Yu. V. Shvyd'ko, Phys. Rev. B 59, 9132 (1999).

[12] J. P. Hannon and G. T. Trammell, Phys. Rev. 169, 315 (1968); 186, 306 (1969); W. Sturhahn and E. Gerdau, Phys. Rev. B 49, 9285 (1994).

[13] A detailed account of the derivation is beyond the scope of this Letter and will be given in a separate publication.

[14] Note that the dependence of $\mathbf{N}$ on $\vec{R}$ does not violate the previous assumption of the sample to be homogeneous, because $\vec{k}_{0} \cdot \vec{R}=$ const along the direction of $\vec{k}_{0}$; see Fig. 1 .

[15] $\overrightarrow{\mathbf{S}}$ leads to a rotation of the polarization vectors and may introduce energy shifts of the nuclear levels in the order of $\hbar \Omega$, see I. Bialynicki-Birula and Z. Bialynicka-Birula, Phys. Rev. Lett. 78, 2539 (1997). The present experiment is not sensitive to such effects since $\hbar \Omega$ is very small compared to the natural linewidth.

[16] Note that the series expansion for $\vec{k}(t)$ is the solution of the equation of motion for a vector precessing with constant angular velocity: $d \vec{k} / d t=\vec{\Omega} \times \vec{k}$ with $\vec{k}(0)=\vec{k}_{0}$.

[17] R. Röhlsberger, E. Gerdau, R. Rüffer, W. Sturhahn, T. S. Toellner, A. I. Churmakov, and E. E. Alp, Nucl. Instrum. Methods Phys. Res., Sect. A 394, 251 (1997).

[18] J. W. Goodman, Statistical Optics (John Wiley, New York, 1985).

[19] E. Gluskin, E.E. Alp, I. McNulty, W. Sturhahn, and J. Sutter, J. Synchrotron Radiat. 6, 1065 (1999).

[20] R. Röhlsberger et al. (to be published).

[21] T. Mooney, T. S. Toellner, W. Sturhahn, E. E. Alp, and S. D. Shastri, Nucl. Instrum. Methods Phys. Res., Sect. A 347, 348 (1994).

[22] A. Q. R. Baron, A. I. Chumakov, H.F. Grünsteudel, H. Grünsteudel, L. Niesen, and R. Rüffer, Phys. Rev. Lett. 77, 4808 (1996). 